\title{
Reduction of travel times and traffic emissions using model predictive control ${ }_{-}^{*}$
}

\author{
S.K. Zegeye, B. De Schutter, H. Hellendoorn, and E. Breunesse \\ If you want to cite this report, please use the following reference instead: \\ S.K. Zegeye, B. De Schutter, H. Hellendoorn, and E. Breunesse, "Reduction of travel \\ times and traffic emissions using model predictive control," Proceedings of the 2009 \\ American Control Conference, St. Louis, Missouri, pp. 5392-5397, June 2009.
}

Delft Center for Systems and Control

Delft University of Technology

Mekelweg 2, 2628 CD Delft

The Netherlands

phone: +31-15-278.24.73 (secretary)

URL: https: //www.dcsc.tudelft.nl

*This report can also be downloaded viahttps://pub. deschutter.info/abs/09_004.html 


\title{
Reduction of Travel Times and Traffic Emissions Using Model Predictive Control
}

\author{
Solomon Kidane Zegeye, Bart De Schutter, Hans Hellendoorn, and Ewald Breunesse
}

\begin{abstract}
In this paper we present a model-based traffic flow control approach to improve both traffic flow and emissions in a traffic network. A model predictive control (MPC) is implemented using a microscopic car-following traffic flow model and an average-speed-based emission model. We consider reduction of total time spent (TTS) and total emissions (TE) as performance measures of the control strategy. Moreover, with the help of simulations we illustrate that a traffic control strategy, particularly an MPC strategy, aiming at the reduction of the TTS does not necessarily reduce the level of emissions. In particular, when the traffic flow is congested, we demonstrate that a traffic control strategy that addresses TTS (or improvement of the traffic flow) alone can cause an increment in the level of emissions and vice versa. Therefore, in this paper we explain how to integrate both requirements so that a balanced trade-off is obtained.
\end{abstract}

\section{INTRODUCTION}

Despite the improvements in transportation systems, the rise of fuel prices, and the imposition of more stringent environmental policies for emission levels, the demand for mobility and transportation is continuously increasing. Consequently roads are frequently congested, creating economical, social, and ecological challenges. Moreover, in recent epidemiological studies of the effects of combustionrelated (mainly traffic-generated) air pollution, $\mathrm{NO}_{2}$ was shown to be associated with adverse health effects [23], [25]. Furthermore, road traffic exhaust emissions account for $40 \%$ of volatile organic compounds, more than $70 \%$ of $\mathrm{NO}_{x}$, and over $90 \%$ of $\mathrm{CO}$ in most European cities [23], and about $45 \%$ of the pollutants released in the US [20]. Frequent and longer congested traffic conditions make this even worse.

There are several possible approaches to address these problems. Large-scale substitution of fossil oil by alternative fuels is a possible solution, but it is not feasible to realize this in the short to medium term. A second possible solution is enhancing vehicle technology. However, vehicle improvements seem to be approaching their limit [17] and they alone cannot solve the problems. Furthermore, the limitations in the availability of land, and economical and environmental constraints often make extending infrastructures infeasible. An alternative and promising solution is the implementation of intelligent transportation systems [24], [26]. Different

S. K. Zegeye, B. De Schutter, and J. Hellendoorn are with Delft Center for Systems and Control, Delft University of Technology, Delft, The Netherlands. B. De Schutter is also with the Marine \& Transport Technology of Delft University of Technology. E. A. Breunesse is with Shell Nederland B. V. The Hague, The Netherlands. $\{\mathrm{s} . \mathrm{k}$. zegeye, j.hellendoorn $\}$ atudelft.nl, b@deschutter.info, ewald.breunessedshell.com traffic flow control measures (such as traffic signal, ramp metering, speed control, route guidance, etc.) can then be used to minimize the impact of traffic jams (such as longer travel times and emissions).

To the best of our knowledge, there are not many papers in the traffic control literature that explicitly aim at the reduction of emissions directly. Many papers either study the effect of different traffic assignment solutions on emissions and fuel consumption [1], [8], [9] or deal with traffic control problems to improve traffic flow [14], [26]. Most traffic control papers address problems related to the reduction of congestion, improving safety, reducing total time travel, and the like. As an example, Hegyi et al. [14] showed that integration of speed limit control and ramp metering can be used to reduce the total time spent (TTS). Related work by Zhang et al. [26] but using microscopic models shows similar results. But, both studies focus on the improvement of traffic flow. However, improvement in traffic flow does not necessarily guarantee reduced emission levels. As will be shown in this paper, a controller that focuses only on reduction of the TTS may result in higher emissions than a controller that also takes emissions into account. This paper shows how to combine the requirements of reducing emissions and travel time so that a balanced trade-off is obtained.

In this paper we use a model-based control approach to reduce emissions while still improving the traffic flow. In particular, we implement Model Predictive Control (MPC) using a car-following model and an average-speed-based emission model. We use speed limit control to control a freeway network to improve the TTS and the total emissions (TE).

The paper starts by discussing both the traffic and the emission models considered in this study in Section II. In Section III the MPC control strategy is presented. Section IV presents a simple case study that illustrates the proposed approach. Finally, Section V gives the conclusions drawn from the work and presents some topics for future work.

\section{MODELS}

\section{A. Traffic flow models}

Traffic flow models can be divided into three classes, viz. macroscopic, microscopic, and mesoscopic [16]. Macroscopic traffic models deal with the average traffic variables (such as average speed, density, and flow). On the other hand microscopic traffic models describe the behavior of individual vehicles in the traffic flow. The position, speed, and acceleration of each vehicle are the states of such 
models. Mesoscopic traffic models describe the behavior of each vehicle (microscopically) using macroscopic variables (such as link flows and link travel times). In other words mesoscopic models combine characteristics of both microscopic and macroscopic traffic flow models. For this study we use a microscopic traffic model, in particular a car-following model. Note that in this paper only the longitudinal kinematic behavior of vehicles and drivers is considered. However, the proposed approach is generic and also valid for other more complex models that also include lane changing behavior.

\section{Vehicle kinematics}

The general longitudinal kinematic motion of the vehicles after discretization is described by:

$$
\begin{aligned}
& x_{i}(\ell+1)=x_{i}(\ell)+v_{i}(\ell) t_{\mathrm{s}}+0.5 a_{i}(\ell) t_{\mathrm{s}}^{2} \\
& v_{i}(\ell+1)=v_{i}(\ell)+a_{i}(\ell) t_{\mathrm{s}}
\end{aligned}
$$

where $x_{i}(\ell), v_{i}(\ell)$, and $a_{i}(\ell)$ are respectively the position, speed, and acceleration of $i^{\text {th }}$ vehicle in the network at time $t=\ell \cdot t_{\mathrm{s}}$, where $\ell$ is the simulation time step counter, while $t_{\mathrm{s}}\left(\right.$ e.g. $\left.t_{\mathrm{s}}=1 \mathrm{~s}\right)$ is the simulation time step of the discretized model. The acceleration in (1)-(2) is determined from the driver model described in the sequel. Moreover, the acceleration is saturated between minimum and maximum acceptable accelerations $a_{\min }$ and $a_{\max }$.

\section{Longitudinal human driver behavior}

The speed and nature of the reaction of drivers is dependent on their headway time (or distance). The time headway is defined as the time difference between two consecutive vehicles that pass a certain location. This can be described as the time needed by the following vehicle to reach the current position of the leading vehicle with its current speed. Mathematically this can be expressed as:

$$
t_{\mathrm{h}}(\ell)=\frac{x_{\mathrm{l}}(\ell)-x_{\mathrm{f}}(\ell)}{v_{\mathrm{f}}(\ell)}
$$

where $x_{1}, x_{\mathrm{f}}$ are the positions of the leading and the following vehicles respectively, and $v_{\mathrm{f}}$ is the speed of the following vehicle.

Depending on the time headway a vehicle can be either in car-following or free-flow mode. When the time headway is larger than the threshold time headway $t_{\mathrm{tr}}$ (e.g., $t_{\mathrm{tr}}=10 \mathrm{~s}$ ), then the vehicle is said to be in free-flow mode. Whereas if the time headway is smaller than the threshold time headway, then the vehicle is in a car-following mode.

In free-flow driving conditions the acceleration of a vehicle is determined by a constant multiple of the difference in the delayed reference speed (or speed limit) and delayed speed of the vehicle. Mathematically, this is described as:

$$
a_{i}(\ell)=F\left(v_{\mathrm{ref}, i}(\ell-\sigma)-v_{i}(\ell-\sigma)\right)
$$

where $F$ is a model parameter (typically 0.01-0.4), $v_{\text {ref }, i}$ is the speed limit (or reference speed) of the $i^{\text {th }}$ vehicle, $\sigma$ is the reaction delay ${ }^{1}$ of the driver. In the car-following

${ }^{1}$ We assume that the reaction delay is an integer multiple of simulation time step. driving mode, where the time headway is smaller than the threshold time headway $t_{\mathrm{tr}}$, the acceleration of the vehicle is determined using car-following models. There are various types of car-following models. A review of various carfollowing models can be found in Brackstone \& McDonald [6]. In this paper we use the Gazis-Herman-Rothery (GHR) [13] stimuli-response car-following model $^{2}$. In this model the reaction of the driver (in other words the acceleration of the vehicle) varies with the variation of its current speed, and the relative speed and position of the vehicle with respect to its predecessor vehicle [4], [6], [16]. The model also takes into account the delay in the reaction of the driver in the relative speed and position of the vehicle.

$$
a_{\mathrm{f}}(\ell)=\alpha v_{\mathrm{f}}^{\beta}(\ell) \frac{\left(v_{\mathrm{l}}(\ell-d)-v_{\mathrm{f}}(\ell-d)\right)}{\left(x_{\mathrm{l}}(\ell-d)-x_{\mathrm{f}}(\ell-d)\right)^{\gamma}}
$$

where $\alpha, \beta$, and $\gamma$ are model parameters, and $d$ is the reaction delay of the driver.

\section{B. Traffic emission models}

Traffic emission models calculate the emissions produced by vehicles based on the operating conditions of the vehicles. Emissions of a vehicle are influenced by the vehicle technology, vehicle status (such as age, maintenance, etc.), vehicle operating conditions, the characteristics of the infrastructure, and external environment conditions. For a given vehicle technology and status of a vehicle, emission models can be calibrated for every vehicle type, or homogeneous vehicle categories. The main inputs to the emission models are the operating conditions of the vehicle (such as speed, acceleration, engine load) [15]. Emission models can be either technology-based engineering models or traffic emission models.

Technology-based emission or fuel consumption models are very detailed models. These kind of models are developed for a specific vehicle (or engine) model [15]. Such models are used for the assessment of new technological developments, and for regulation purposes [15]. Since these models are very detailed, they are difficult to use for online prediction or on-line estimation of emissions and fuel consumption of traffic flow. Therefore, for computational reasons it is advisable not to use such models for on-line model-based traffic control purposes.

Traffic emission models are more simple, and they are developed for diverse collections of vehicles grouped in homogeneous categories. In general these models are calibrated based on the operating conditions of the vehicle in a traffic flow. Traffic emission models can be either average-speed-based or dynamic. For its simplicity of use, and for being a long established method [5] we have used (a dynamic version of) an average-speed-based model for this study. In principle the input for an average-speed-based model is the trip-based average speed, i.e. the average speed of a vehicle for a complete trip is used to compute the

\footnotetext{
${ }^{2}$ Other models are also possible (e.g. CTM [10] or METANET [19]).
} 


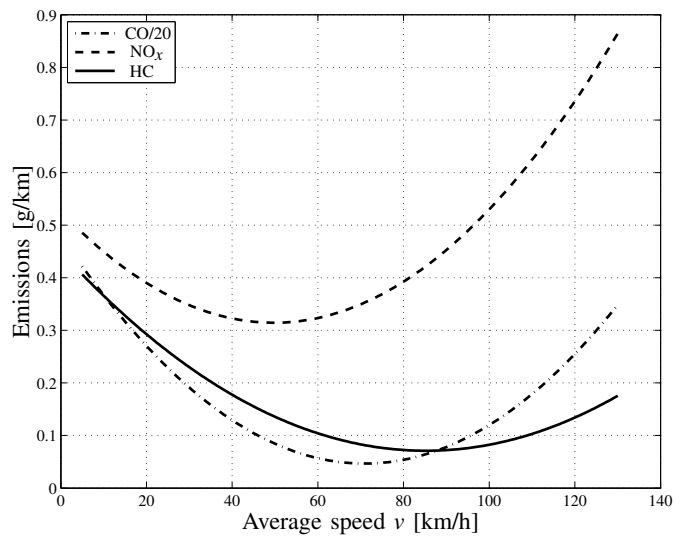

(a) Emissions in $\mathrm{g} / \mathrm{km}$

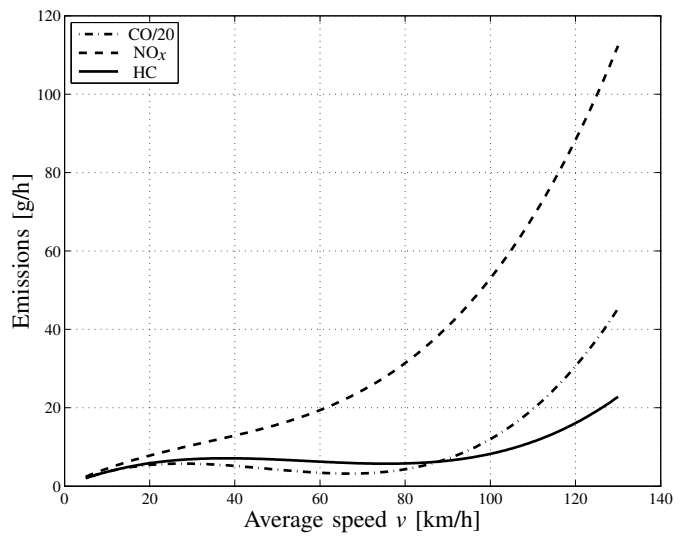

(b) Emissions in $\mathrm{g} / \mathrm{h}$

Fig. 1. Average speed based emission model of [5].

emission rate of the vehicle. But in practice it is also common to use local speed inputs [5]. In this approach, the speed of the vehicle at each time instant can be used to estimate the emission rate of the vehicle at that time instant. Clearly, using the local speeds to compute the emission rates can give more accurate results. This is for the simple reason that by using local speed inputs, it is possible to capture the transient behavior of the vehicle in estimating the emission rates. This approach implicitly includes the acceleration of a vehicle in estimating the emission rates. Therefore, we also use the local speed input approach in our emission models.

The emission model considered for this study is obtained from COPERT III [22]. Fig. 1(a) shows the model used for $\mathrm{CO}, \mathrm{NO}_{x}$, and $\mathrm{HC}$ emissions. The mathematical expressions for this model of each of the emissions are:

$$
\begin{aligned}
& E_{\mathrm{CO}}(v)=\left(0.001728 v^{2}-0.245 v+9.617\right)[\mathrm{g} / \mathrm{km}] \\
& E_{\mathrm{NO}_{x}}(v)=10^{-4}\left(0.854 v^{2}-85 v+5260\right)[\mathrm{g} / \mathrm{km}] \\
& E_{\mathrm{HC}}(v)=10^{-4}\left(0.521 v^{2}-88.8 v+4494\right)[\mathrm{g} / \mathrm{km}]
\end{aligned}
$$

where $v$ is the average speed and $E_{\mathrm{CO}}, E_{\mathrm{NO}_{x}}$ and $E_{\mathrm{HC}}$ denote the emission levels of carbon monoxide $(\mathrm{CO})$, oxides of nitrogen $\left(\mathrm{NO}_{x}\right)$, and hydrocarbons $(\mathrm{HC})$ in grams per kilometer respectively.

As the output of the emission model in (6)-(8) is given in $\mathrm{g} / \mathrm{km}$, we change the expressions into emission functions that give emissions in $\mathrm{g} / \mathrm{h}$. This is done by multiplying the expressions (6)-(8) by the average speed $v$. This model makes computation of emission levels of each vehicle simpler. The plots of the transformed equations are given in Fig. 1(b). We can get the emission levels at each simulation step by multiplying the output of the model with the simulation time step. This model can then be used to get second-by-second emissions of a vehicle in a network. The new expressions of the model have a structure that is similar to the model of Ahn et al. [2] when the acceleration is zero.

\section{MODEL PREDICTIVE CONTROL}

\section{A. Philosophy of model predictive control}

The basic concept of Model Predictive Control (MPC) [7], [18] lies in the optimization of control inputs based on prediction and a moving horizon approaches. MPC uses an on-line optimization method, based on the measurement of current and future predicted evolution of the system states. Using a model of the system and numerical optimization, it determines a sequence of control inputs that optimize a performance criterion over a given future time horizon (i.e. from control step $k$ up to $k+N_{\mathrm{p}}$ ). However, only the first control input is applied for the system in a moving horizon concept. i.e. at each control time step only the first sample of the optimal control input is applied to the system; afterward the time axis is shifted one control sample time step. Then, based on the new states and control inputs of the system, new sequence of optimal control inputs are generated. Ones again the first control input is applied. At every time step the process in repeated. This process is repeated until the end of the simulation time.

Fig. 2(a) illustrates the interrelationship of the traffic system and MPC controller, and Fig. 2(b) depicts the concepts of prediction and control horizons. We consider both the traffic system and MPC controller in discrete time. Recall that $t_{\mathrm{s}}$ represents the simulation time. We define the control time step $t_{\mathrm{c}}$ (a typically value is $t_{\mathrm{c}}=1 \mathrm{~min}$ ). For the sake of simplicity we assume that $t_{\mathrm{c}}=M t_{\mathrm{s}}$, for some positive integer $M$. Therefore, at time $t=k \cdot t_{\mathrm{c}}=\ell \cdot t_{\mathrm{s}}$ the controller time step counter $k$ is an integer divisor of the simulation time counter $\ell$. They are related by $\ell(k)=M k$. A measurement of the traffic states (such as position, speed, acceleration, etc.) is made every $t_{\mathrm{c}}$ time units and the traffic control measures (such as speed limits, ramp metering rates, etc.) will be applied for the next $t_{\mathrm{c}}$ time units (see Fig. 2(a)). In other words, after a control signal is applied for $M$ sample steps of $t_{\mathrm{s}}$ time units, a new measurement of the states of the traffic system is undertaken and the MPC controller generates and applies new control inputs by predicting the evolution of the system states from the current time $t=k \cdot t_{\mathrm{c}}$ up to $t=\left(k+N_{\mathrm{p}}\right) \cdot t_{\mathrm{c}}$ (see Fig. 2(b)).

The main advantage of MPC is its ability to take constraints into account and that it can be used for nonlinear systems. Its main limitation emanates from the computation time required by the optimization process. To alleviate the 
computational problems several methods can be used (e.g. introducing control horizon). In order to limit the number of variables to be optimized, thereby to improve computation speed, a control horizon $N_{\mathrm{c}} \leq N_{\mathrm{p}}$ is defined after which the control input is kept constant, i.e. $u(k+j)=u(k+j-1)$ for $j=N_{\mathrm{c}}, \ldots, N_{\mathrm{p}}-1$, where $N_{\mathrm{c}} \leq N_{\mathrm{p}}$.

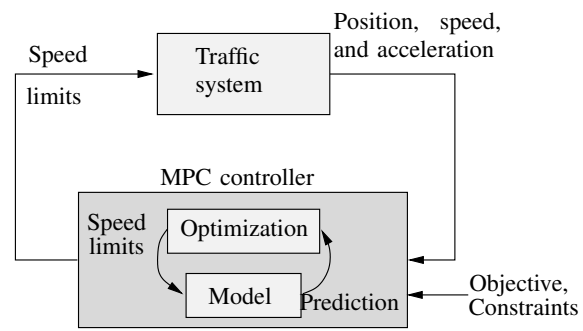

(a) Schematic representation

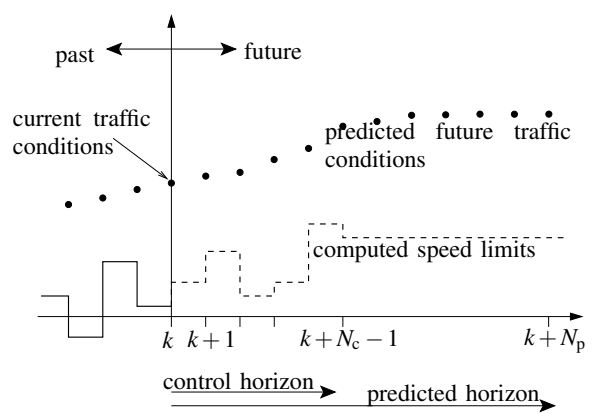

(b) Prediction and control horizon

Fig. 2. Conceptual representation of model predictive control.

\section{B. MPC for traffic and emission control}

Besides the difference in the effects of traffic speed on emissions and on total time spent, the minimum of the traffic emissions are attained at different traffic speeds. This makes it difficult to decide which speed limits to select to optimally reduce the level of the emissions. Reducing the total emissions may have more influence on some gases than others. In the WHO [25] report it is shown that $\mathrm{NO}_{x}$ has a stronger adverse health effect than the other gases. However, gases like $\mathrm{CO}$ have adverse effect in the long run. By assigning relative weight (policies) to the different emissions and the TTS it is possible to use a model-based traffic control to set the optimal speed limits which will result in a balanced trade-off of the conflicting requirements.

In this study we use an MPC controller to control the traffic flow using speed limits. We investigate the impact of speed limit control on the improvement of the total time spent (TTS) and the total emissions (TE) in a traffic network. The model of the optimization accommodates both a traffic flow model and an emission model. As prediction model we will use the models presented in Section II. However, note that the MPC approach is generic and can also accommodate other, more complex traffic flow and emission models [2], [19].

At a control time step $k$, the MPC controller predicts the evolution of the traffic flow and the emission levels in the network over the time interval $\left[k t_{\mathrm{c}},\left(k+N_{\mathrm{p}}\right) t_{\mathrm{c}}\right)$ and it optimizes the speed limit control sequence $u(k), u(k+$ $1), \ldots, u\left(k+N_{\mathrm{c}}-1\right)$ in such a way that the objective function is reduced. After the optimal control input sequence $u^{*}(k), u^{*}(k+1), \ldots, u^{*}\left(k+N_{\mathrm{c}}-1\right)$ has been computed, the first sample $u^{*}(k)$ is applied to the system until the next control step $k+1$. Subsequently, whole process is repeated all aver again.

As objective function we could for example consider the following expression ${ }^{3}$ :

$$
\begin{aligned}
& J(k)=\frac{\lambda_{1}}{\mathrm{TTS}_{\text {nominal }}} \sum_{j=1}^{M N_{\mathrm{p}}} \mathscr{N}(\ell(k)+j) t_{\mathrm{s}} \\
& +\frac{\lambda_{2} \mu_{1}}{\mathrm{TE}_{\mathrm{CO}, \text { nominal }}} \sum_{j=1}^{M N_{\mathrm{p}}} \sum_{i \in \mathscr{V}(\ell(k)+j)} E_{\mathrm{CO}}\left(v_{i}(\ell(k)+j)\right) v_{i}(\ell(k)+j) t_{\mathrm{s}} \\
& +\frac{\lambda_{2} \mu_{2}}{\mathrm{TE}_{\mathrm{NO}_{x}, \text { nominal }}} \sum_{j=1}^{M N_{\mathrm{p}}} \sum_{i \in \mathscr{V}(\ell(k)+j)} E_{\mathrm{NO}_{x}}\left(v_{i}(\ell(k)+j)\right) v_{i}(\ell(k)+j) t_{\mathrm{s}} \\
& +\frac{\lambda_{2} \mu_{3}}{\mathrm{TE}_{\mathrm{HC}, \text { nominal }}} \sum_{j=1}^{M N_{\mathrm{p}}} \sum_{i \in \mathscr{V}(\ell(k)+j)} E_{\mathrm{HC}}\left(v_{i}(\ell(k)+j)\right) v_{i}(\ell(k)+j) t_{\mathrm{s}} \\
& +\frac{\lambda_{3}}{{\mathrm{~T} \Delta u_{\mathrm{nominal}}}_{N_{\mathrm{c}}-1}^{N_{j=0}}\|u(k+j)-u(k+j-1)\|_{2}^{2}}
\end{aligned}
$$

where, $\lambda_{n} \geq 0$, and $\mu_{n} \geq 0$ for $n=1,2,3$ are weighting coefficients, $\mathscr{N}(\ell)$ denotes the number of vehicles in the network (and the queues) at time $t=\ell t_{\mathrm{s}}$, and $\mathscr{V}(\ell)$ denotes the set of vehicles present in the network at time $t=\ell t_{\mathrm{s}}$. Note that each term in the objective function contains a normalization factor consisting of a "nominal" value for respectively the total time spent $\left(\mathrm{TTS}_{\text {nominal }}\right)$, the total $\mathrm{CO}$ emission $\left(\mathrm{TE}_{\mathrm{CO} \text {,nominal }}\right)$, the total $\mathrm{NO}_{x}$ emission $\left(\mathrm{TE}_{\mathrm{NO}_{x}, \text { nominal }}\right)$, the total $\mathrm{HC}$ emission $\left(\mathrm{TE}_{\mathrm{HC}, \text { nominal }}\right)$, and a measure for the total speed limit difference ( $\left.\mathrm{T} \Delta u_{\text {nominal }}\right)$ (see also Section IV-B for an example of how to compute these normalization factors). Moreover, in practice the values of the weights $\lambda_{n}$ and $\mu_{n}$ are obtained by the traffic management authorities or by the traffic policies.

\section{Optimization method}

One of the bottlenecks in MPC control approach is the extensive optimization and the resulting computational requirements. The MPC optimization problem considered for this study is nonlinear and nonconvex. Thus a proper choice of an optimization technique has to be made in order to obtain feasible optimal control values. Owing to the nonconvex nature of the objective function, global, or multi-start local optimization methods are required. Hence, multi-start sequential quadratic programming [21], pattern search [3], generic algorithms [11], or simulated annealing [12] can be used.

\footnotetext{
${ }^{3}$ Note however that MPC is generic as regards the choice of the performance criteria, and so other objective functions could also be considered instead.
} 


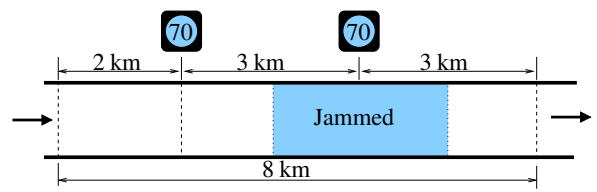

Fig. 3. Layout of the case study.

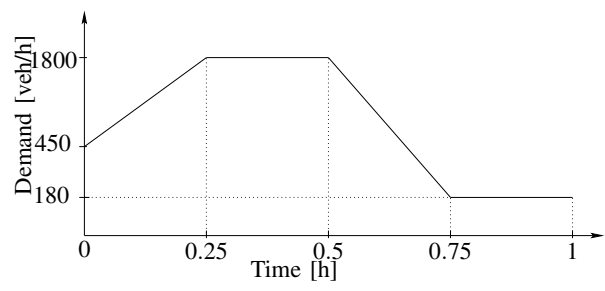

Fig. 4. Demand profile.

\section{CASE STUDY}

In this section we demonstrate the applicability of the MPC traffic control strategy mentioned in Section III on a simple case study. We consider this simulation benchmark to investigate the effect of the control strategy. The layout of the freeway, the performance criterion, and the simulation results are given in the subsequent subsections.

\section{A. Traffic freeway layout}

We consider a single-lane one-way $8 \mathrm{~km}$ freeway. As depicted in Fig. 3, the roadway is divided into three sections, and two speed limit controls are applied in the last two sections. We consider an experiment that starts at $t_{\text {start }}=0 \mathrm{~h}$, and ends at $t_{\text {end }}=1 \mathrm{~h}$. At the starting time $t_{\text {start }}$ the segment of the freeway from $3.5 \mathrm{~km}$ to $6.5 \mathrm{~km}$ is assumed to be congested. The traffic demand varies over the whole span of the simulation time (see Fig. 4). We have considered the same demand for all the different cases considered in this study. Moreover, the parameters that we have used for the MPC controllers are tabulated in Table I.

\section{B. Performance criterion}

In this case study we have considered the performance criterion defined in (9). The normalization factors

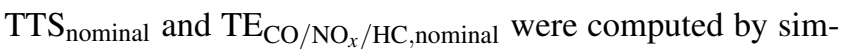
ulating the traffic system for the $8 \mathrm{~km}$ freeway with a speed

TABLE I

MPC CONTROLLER PARAMETERS

\begin{tabular}{l|l|l} 
MPC Parameters & Values & Remarks \\
\hline \hline$T_{\text {sim }}$ & $60 \mathrm{~min}$ & Simulation time \\
$N_{\mathrm{p}}$ & $10 \mathrm{~min}$ & Prediction horizon \\
$N_{\mathrm{c}}$ & $2 \mathrm{~min}$ & Control horizon \\
$t_{\mathrm{c}}$ & $1 \mathrm{~min}$ & Control time step \\
$t_{\mathrm{S}}$ & $1 \mathrm{~s}$ & Simulation time step \\
$v_{\max }$ & $120 \mathrm{~km} / \mathrm{h}$ & Maximum speed limit \\
$v_{\min }$ & $40 \mathrm{~km} / \mathrm{h}$ & Minimum speed limit
\end{tabular}

TABLE II

WEIGHTING CONSTANTS FOR DIFFERENT CASES

\begin{tabular}{c||c|c|c||c|c||}
\cline { 2 - 6 } \multicolumn{1}{c|}{ Cases } & \multicolumn{3}{c||}{ Weighting } & \multicolumn{2}{c||}{ Simulation results } \\
\cline { 2 - 6 } & $\lambda_{1}$ & $\lambda_{2}\left(\mu_{1}, \mu_{2}, \mu_{3}\right)$ & $\lambda_{3}$ & $\begin{array}{c}\text { TTS } \\
{[\mathrm{veh} \cdot \mathrm{h}]}\end{array}$ & $\begin{array}{c}\text { Total emissions } \\
{[\mathrm{kg}]}\end{array}$ \\
\hline \hline case 1 & 0 & 0 & 0 & 383.1 & 13.24 \\
case 2 & 1 & 0 & 0.01 & 210.4 & 14.92 \\
case 3 & 0 & $1(1,1,1)$ & 0.01 & 340.6 & 8.29 \\
case 4 & 1 & $1(1,1,1)$ & 0.01 & 340.2 & 8.27 \\
\hline
\end{tabular}

limit of $80 \mathrm{~km} / \mathrm{h}$ and for the scenario given in Section IVA. A value for $\mathrm{T} \Delta u_{\text {nominal }}$ is computed as follows: we consider a simulation where the speed limit changes with $v_{\text {step }}=10 \mathrm{~km} / \mathrm{h}$ at every control step. So

$$
\mathrm{T} \Delta u_{\text {nominal }}=\sum_{k=0}^{N_{\mathrm{c}}-1} v_{\text {step }}^{2}=N_{\mathrm{c}} v_{\text {step }}^{2}
$$

We have considered different weighting values to analyze the effects of different control policies on emissions and traffic flow. The combinations considered in this study are given in Table II.

For solving the MPC optimization problem we have adopted a multi-start sequential quadratic programming (SQP) [21] optimization method. More specifically, we have used the fmincon command of the Matlab optimization toolbox.

\section{Simulation results}

Two performance measures are defined to analyze the simulation results, viz. total time spent (TTS) and total emissions (TE). The TTS is the sum total of the time spent by all vehicles in the network. The TE is the sum total of particular emission type of all vehicles in the network. The system has been simulated for $1 \mathrm{~h}$ of real simulation time. This has been done for uncontrolled and controlled scenarios. The results of the simulation are shown in Table II.

As it can be seen from the table, the TTS and the TE are $383.1 \mathrm{veh} \cdot \mathrm{h}$ and $13.24 \mathrm{~kg}$ respectively when the system is not controlled (case 1). When an MPC controller with an objective function of reducing TTS (case 2) is used, the TTS is reduced by $45.1 \%$, while the TE is increased by $12.68 \%$. With the same controller, but with an objective of reducing emissions (case 3), the TTS is reduced by $11.23 \%$ and the TE is reduced by $37.39 \%$. The TTS in case 3 is larger than the TTS in case 2. This shows that controlling the total time spent or the total emissions alone has negative effects on emissions and traffic flow respectively. Neither does a reduction in TTS imply reduced emissions nor does a reduction in total emissions imply a reduced TTS.

In case 4, the weighted sum of TTS and TE is considered as a cost function of the optimization in the MPC controller. The change in TTS and TE is insignificant relative to case 3. But case 4 offers significant improvements in regard to TE as compared to case 1 and case 2 . The changes are also 
more noticeable when the weighting factors are changed. Moreover, the controller with an objective of reducing TTS and TE reduces the TTS by $11.23 \%$ (compare case 1 and case 4 ) and the TE by $37.53 \%$.

The results indicate that for the given case study the objective of reducing emissions and TTS are two conflicting requirements. It is difficult to get lowest emissions without restricting the traffic flow or vice versa. This indicates that higher flows (or speeds) do not guarantee reduced emissions. Thus, MPC can offer a balanced trade-off due to weights in the MPC cost criterion (see (9)).

\section{CONCLUSIONS AND FUTURE WORK}

We have proposed a model-based traffic flow control approach to reduce both total emissions and total time spent. This control method uses a prediction model and online optimization to determine the optimal traffic control measures over a given prediction horizon, which are subsequently applied using a receding horizon approach. We have illustrated the approach using a car-following traffic flow model and a dynamic version of an average-speedbased emission model. In addition, we have considered a case study involving a single-lane one-way traffic freeway to show how MPC can be applied to provide a balanced tradeoff between conflicting performance measures. The results of this case study also demonstrate the possible solutions MPC can offer for mobility and environmental challenges.

We have discussed the possible conflicting requirements of the demand for transportation and the environmental constraints. Based on simulation results, we have shown that the focus on the reduction of total time spent (TTS) alone cannot meet the requirement of reducing emissions. The simulation results suggest that both emission reduction and traffic flow improvement can also be attained by proper definition of the objective function of the MPC controller. More specifically, a 37.53\% reduction of total emission (TE) and $11.23 \%$ reduction of TTS has been obtained for the simulation study.

In future work we will consider more complex case studies, investigate implementation of constraints on the emission levels, and integration of speed limit control and ramp metering for the reduction of emissions and of the total time spent. We will use other more detailed nonlinear traffic flow models (such as CTM [10] or METANET [19]). Furthermore, we will compare different traffic control approaches.

\section{ACKNOWLEDGMENTS}

This research is supported by the Shell/TU Delft Sustainable Mobility program, the BSIK project Transition towards Sustainable Mobility (TRANSUMO), the Transport Research Center Delft, and the European COST Action TU0702.

\section{REFERENCES}

[1] K. Ahn and H. Rakha. The effects of route choice decisions on vehicle energy consumption and emissions. Transportation Research Part D, 13(3):151-167, May 2008.

[2] K. Ahn, A. A. Trani, H. Rakha, and M. Van Aerde. Microscopic fuel consumption and emission models. In Proceedings of the $78^{\text {th }}$ Annual Meeting of the Transportation Research Board, Washington DC, USA, January 1999. CD-ROM.

[3] C. Audet and J. E. Dennis Jr. Analysis of generalized pattern searches. SIAM Journal on Optimization, 13(3):889-903, 2007.

[4] C. S. Bong and S. K. Han. Development of sensitivity term in car-following model considering practical driving behavior of preventing rear end collisions. Journal of the Eastern Asia Society for Transportation Studies, 6:1354-1367, 2005.

[5] P. G. Boulter, T. Barlow, I. S. McCrae, S. Latham, D. Elst, and E. van der Burgwal. Road traffic characteristics, driving patterns and emission factors for congested situations. Technical report, TNO Automotive, Department Powertrains-Environmental Studies \& Testing, Delft, The Netherlands, 2002. OSCAR Deliverable 5.2.

[6] M. Brackstone and M. McDonald. Car-following: a historical review. Transportation Research Part F, 2(4):181-196, 2000.

[7] E. F. Camacho and C. Bordons. Model Predictive Control in the Process Industry. Springer-Verlag, Berlin, Germany, 1995.

[8] M. C. Coelho, T. L. Farias, and N. M. Rouphail. Impact of speed control traffic signals on pollutant emissions. Transportation Research Part D, 10(4):323-340, July 2005.

[9] M. C. Coelho, T. L. Farias, and N. M. Rouphail. Effect of roundabout operations on pollutant emissions. Transportation Research Part D, 11(5):333-343, September 2006.

[10] C. F. Daganzo. The cell transmission model: A dynamic representation of highway traffic consistent with the hydrodynamic. Transportation Research Part B, 28B(4):269-287, August 1994.

[11] L. Davis, editor. Handbook of Genetic Algorithms. Van Nostrand Reinhold, New York, USA, 1991

[12] R. W. Eglese. Simulated annealing: A tool for operations research. European Journal of Operational Research, 46(3):271-281, 1990.

[13] D. Gazis, R. Herman, and R. Rothery. Nonlinear follow the leader models of traffic flow. Operations Research, 9(4):545-567, 1961.

[14] A. Hegyi, B. De Schutter, and H. Hellendoorn. Model predictive control for optimal coordination of ramp metering and variable speed limits. Transportation Research Part C, 13(3):185-209, June 2005.

[15] J. Heywood. Internal Combustion Engine Fundamentals. McGrawHill, New York, 1988.

[16] S. P. Hoogendoorn and P. H. L. Bovy. State-of-the-art of vehicular traffic flow modelling. Proceedings of the Institution of Mechanical Engineers, Part I: Journal of Systems and Control Engineering, 215(4):283-303, 2001.

[17] Y. Kishi, S. Katsuki, Y. Yoshikawa, and I. Morita. A method for estimating traffic flow fuel consumption-using traffic simulations. The Society of Automotive Engineers of Japan Review, 17(3):307-311, July 1996.

[18] J. M. Maciejowski. Predictive Control with Constraints. Prentice Hall, Harlow, England, 2002.

[19] A. Messmer and M. Papageorgiou. METANET: A macroscopic simulation program for motorway networks. Traffic Engineering and Control, 31(9):466-470, 1990.

[20] NRC. Expanding metropolitan highways: Implications for air quality and energy use. Technical report, National Academy Press, Washington DC, USA, 1995.

[21] P. M. Pardalos and M. G. C. Resende. Handbook of Applied Optimization. Oxford University Press, Oxford, UK, 2002.

[22] Z. Samaras and L. Ntziachristos. Average hot emission factors for passenger cars and light duty trucks. Technical Report 9811, Deliverable 7 of the MEET project, Aristotle National University of Thessaloniki, Greece, June 1998.

[23] S. Schmidt and R. P. Schäfer. An integrated simulation systems for traffic induced air pollution. Environmental Modeling \& Software, 13(3-4):295-303, 1998.

[24] M. van den Berg, A. Hegyi, B. De Schutter, and J. Hellendoorn. A macroscopic traffic flow model for integrated control of freeway and urban traffic networks. In Proceedings of the $42^{\text {nd }}$ IEEE Conference on Decision and Control, pages 2774-2779, Maui, Hawaii USA, December 2003. 
[25] WHO. Health aspects of air pollution, Results from the WHO projects "Systematic review of health aspects of air pollution in Europe". Technical report, World Health Organization, June 2004.

[26] J. Zhang, A. Boiter, and P. Ioannou. Design and evaluation of a roadway controller for freeway traffic. In Proceedings of the $8^{\text {th }}$ International IEEE Conference on Intelligent Transportation Systems, pages 543-548, Vienna, Austria, September 2005. 